\title{
Impact of cooperation uncertainty on the robustness of manufacturing service system
}

\author{
Liang, P.P. ${ }^{a,{ }^{*}}$, Li, C.W..$^{\text {b.* }}$ \\ ${ }^{a}$ School of Economics and Management, Anqing Normal University, Anqing, P.R. China \\ ${ }^{b}$ School of Economics, Huaibei Normal University, Huaibei, P.R. China
}

\section{A B S T R A C T}

The cooperation between enterprises is actually at a certain risk of interruption, which has a significant impact on the robustness of manufacturing service system (MSS). Evaluating MSS' robustness is integral to production and service provisioning, and thus the influence mechanism should be clearly revealed for assisting professionals in the company in improving the robust performance. In this paper, we present an effective methodology for explicating the impact of cooperation uncertainty on the robustness of MSS from a complex system standpoint. This methodology characterizes MSS as a topological network consisting of serval service subsystems, and constructs the measure metrics system of which the validity and applicability are proved theoretically from the dimension of structure and performance. Furthermore, it simulates the cooperation interruption from four different scenarios with algorithms, and finally takes an elevator manufacturing service network as the case to illustrate this novel methodology. The simulation findings suggest that identifying the critical paths in MSS and standardizing the cooperation mechanism within and among core manufacturing service principals outperform the other measures in improving the robustness of MSS.
\end{abstract}

\section{ARTICLE INFO}

Keywords:

Manufacturing service system;

Complex system;

Robustness;

Robustness metric;

Cooperation uncertainty

*Corresponding author:

cwli2008@163.com

(Li, C.W.)

614lpp@163.com

(Liang, P.P.)

Article history:

Received 16 November 2018

Revised 21 April 2019

Accepted 6 May 2019

\section{References}

[1] Zhao, Y., Zhang, W., Liu, D., Bao, F., Tian, L. (2017). Service implementation in manufacturing firms: The role of service-orientated human resource management practices and demand-side search, Management Decision, Vol. 55, No. 4, 648-661, doi: 10.1108/MD-12-2015-0605.

[2] Huang, S., Zeng, S., Fan, Y., Huang, G.Q. (2011). Optimal service selection and composition for service-oriented manufacturing network, International Journal of Computer Integrated Manufacturing, Vol. 24, No. 5, 416-430, doi: $\underline{10.1080 / 0951192 X .2010 .511657 .}$.

[3] Almaktoom, A.T., Krishan, K.K., Wang, P., Alsobhi, S. (2014). Assurance of system service level robustness in complex supply chain networks, International Journal of Advanced Manufacturing Technology, Vol. 74, No. 1-4, 445-460, doi: 10.1007/s00170-014-5883-7.

[4] Buzacott, J.A., Peng, H.S. (2012). Contract design for risk sharing partnerships in manufacturing, European Journal of Operational Research, Vol. 218, No. 3, 656-666, doi: 10.1016/j.ejor.2011.12.008.

[5] Olander, H., Hurmelinna-Laukkanen, P., Blomqvist, K., Ritala, P. (2010). The dynamics of relational and contractual governance mechanisms in knowledge sharing of R\&D projects, Knowledge and Process Management, Vol. 17, No. 4, 188-204, doi: 10.1002/kpm.356.

[6] Zhang, W., Shi, Y., Yang, M., Gu, X., Tang, R., Pan, X. (2017). Ecosystem evolution mechanism of manufacturing service system by service providers, International Journal of Production Research, Vol. 55, No. 12, 3542-3558, doi: 10.1080/00207543.2017.1305131.

[7] Clements, K.C., Stephens, D.W. (1995). Testing models of non-kin cooperation: Mutualism and the Prisoner's Dilemma, Animal Behaviour, Vol. 50, No. 2, 527-535, doi: 10.1006/anbe.1995.0267. 
[8] Schmenner, R.W. (2009). Manufacturing, service and their integration: Some history and theory, International Journal of Operations \& Production Management, Vol. 29, No. 5, 431-443, doi: 10.1108/01443570910953577.

[9] Liang, P., Sun, Y., Pan, Y. (2015). The co-evolution mechanism and stability analysis of service-oriented manufacturing enterprise system, Journal of Industrial Engineering and Management, Vol. 8, No. 5, 1475-1490, doi: 10.3926/jiem.1457.

[10] Smith Ring, P., van de Ven, A.H. (1992). Structuring cooperative relationships between organizations, Strategic Management Journal, Vol. 13, No. 7, 483-498, doi: 10.1002/smj.4250130702.

[11] Cheng, J.-H. (2011). Inter-organizational relationships and knowledge sharing in green supply chains - Moderating by relational benefits and guanxi, Transportation Research Part E: Logistics and Transportation Review, Vol. 47, No. 6, 837-849, doi: 10.1016/i.tre.2010.12.008.

[12] Buston, P.M., Balshine, S. (2007). Cooperating in the face of uncertainty: A consistent framework for understanding the evolution of cooperation, Behavioural Process, Vol. 76, No. 2, 152-159, doi: 10.1016/j.beproc.2007.01.020.

[13] Bilgiç, T., Güllü, R. (2016). Innovation race under revenue and technology uncertainty of heterogeneous firms where the winner does not take all, IIE Transactions, Vol. 48, No. 6, 527-540, doi: 10.1080/0740817X.2015. 1110651.

[14] Chen, Q., Deng, L.F., Wang, H.M. (2018). Optimization of multi-task job-shop scheduling based on uncertainty theory algorithm, International Journal of Simulation Modelling, Vol. 17, No. 3, 543-552, doi: 10.2507/IJSIMM17 (3) $\mathrm{CO} 14$

[15] Hua, Z., Li, S. (2008). Impacts of demand uncertainty on retailer's dominance and manufacturing-retailer supply chain cooperation, Omega, Vol. 36, No. 5, 697-714, doi: 10.1016/i.omega.2006.02.005.

[16] Boukas, E.K. (2006). Manufacturing system: LMI approach, IEEE Transactions on Automatic Control, Vol. 51, No. 6, 1014-1018, doi: 10.1109/TAC.2006.876945.

[17] Huang, X.-Y., Yan, N.-N., Guo, H.-F. (2007). An Ho control method of the bullwhip effect for a class of supply chain system, International Journal of Production Research, Vol. 45, No. 1, 207-226, doi: 10.1080/00207540600678912.

[18] Liu, G.Y., Li, Z.W., Barkaoui, K., Al-Ahmari, A.M. (2013). Robustness of deadlock control for a class of Petri nets with unreliable resources, Information Sciences, Vol. 235, 259-279, doi: 10.1016/ji.ins.2013.01.003.

[19] Mhalla, A., Jerbi, N., Dutilleul, S.C., Craye, E., Benrejeb, M. (2013). Robust control strategies facing disturbances in manufacturing workshops with time constrains, Computers \& Industrial Engineering, Vol. 65, No. 2, 268-276, doi: 10.1016/i.cie.2013.03.003.

[20] Chica, M., Cordón, Ó., Damas, S., Bautista, J. (2013). A robustness information and visualization model for time and space assembly line balancing under uncertain demand, International Journal of Production Economics, Vol. 145, No. 2, 761-772, doi: 10.1016/j.ijpe.2013.05.030.

[21] Boorla, S.M., Howard, T.J. (2016). Production monitoring system for understanding product robustness, Advances in Production Engineering \& Management, Vol. 11, No. 3, 159-172, doi: 10.14743/apem2016.3.217.

[22] Suri, R., Otto, K. (2001). Manufacturing system robustness through integrated modeling, Journal of Mechanical Design, Vol. 123, No. 4, 630-636, doi: 10.1115/1.1402129.

[23] Kusumoto, S., Kitamura, A., Nakamura, M. (2012). Robust scheduling for plural lot size manufacturing, In: Proceedings of ASME/ISCIE International Symposium on Flexible Automation, St. Louis, Missouri, USA, 359-364, doi: 10.1115/ISFA2012-7244.

[24] Mondal, S.C., Ray, P.K., Maiti, J. (2014). Modelling robustness for manufacturing processes: A critical review, International Journal of Production Research, Vol. 52, No. 2, 521-538, doi: 10.1080/00207543.2013.837588.

[25] Watts, D.J., Strogatz, S.H. (1998). Collective dynamics of 'small-world' networks, Nature, Vol. 393, No. 6684, 440442, doi: $10.1038 / 30918$.

[26] Dong, J., Horvath, S. (2007). Understanding network concepts in modules, BMC Systems Biology, Vol. 1, No. 1, Start page: 24 , doi: 10.1186/1752-0509-1-24.

[27] Neumayer, S., Modiano, E. (2010). Network reliability with geographically correlated failures, In: Proceedings of 29th IEEE INFOCOM Conference on Information Communications, San Diego, California, USA, 1658-1666, doi: 10.1109/INFCOM.2010.5461984.

[28] Boorla, S.M., Troldtoft, M.E., Eifler, T., Howard, T.J. (2017). Quantifying the robustness of process manufacturing concept - A medical product case study, Advances in Production Engineering \& Management, Vol. 12, No. 2, 127138, doi: 10.14743/apem2017.2.245.

[29] Wu, J., Mauricio, B., Tan, Y.-J., Deng, H.-Z. (2010). Natural connectivity of complex networks, Chinese Physics Letters, Vol. 27, No. 7, doi: 10.1088/0256-307X/27/7/078902.

[30] Manzano, M., Calle, E., Torres-Padrosa, V., Segovia, J., Harle, D. (2013). Endurance: A new robustness measure for complex networks under multiple failure scenarios, Computer Networks, Vol. 57, No. 17, 3641-3653, doi: 10.1016/j.comnet.2013.08.011. 
APEM
Advances in Production Engineering \& Management

Letnik 14 | Številka 2 | Junij 2019 | Strani 189-200

https://doi.org/10.14743/apem2019.2.321
ISSN 1854-6250

Spletna stran: apem-journal.org Izvirni znanstveni članek

\title{
Vpliv negotovosti sodelovanja na robustnost sistema proizvodnih storitev
}

\author{
Liang, P.P. ${ }^{a,}{ }^{*}$, Li, C.W..$^{\text {b,* }}$ \\ ${ }^{a}$ School of Economics and Management, Anqing Normal University, Anqing, P.R. China \\ ${ }^{b}$ School of Economics, Huaibei Normal University, Huaibei, P.R. China
}

\section{POVZETEK}

Pri sodelovanju med podjetji predstavlja prekinitev sodelovanja tveganje, ki pomembno vpliva na robustnost sistema proizvodnih storitev (MSS). Ocenjevanje robustnosti MSS-jev je sestavni del proizvodnje in zagotavljanja storitev, zato je treba za pomoč strokovnjakom v podjetjih jasno razkriti mehanizme vpliva za izboljšanje robustne zmogljivosti. $\mathrm{V}$ tem prispevku predstavljamo učinkovito metodologijo za izražanje vpliva negotovosti sodelovanja na robustnost MSS s kompleksnega sistemskega stališča. Ta metodologija označuje MSS kot topološko omrežje, sestavljeno iz številnih storitvenih podsistemov in vzpostavi sistem vrednotenja, katerega veljavnost in uporabnost sta teoretično potrjeni z dimenzijo strukture in zmogljivostjo. Za ponazoritev delovanja nove metodologije se $\mathrm{z}$ algoritmom simulira prekinitev sodelovanja na štirih različnih študijskih primerih ter na primeru mreže za proizvodnjo dvigal. Simulacije kažejo, da prepoznavanje kritičnih poti v MSS in standardizacija mehanizma sodelovanja znotraj in med glavnimi proizvodnimi storitvami presegajo druge ukrepe za izboljšanje robustnosti MSS.

(c) 2019 CPE, University of Maribor. All rights reserved.

\section{PODATKI O ČLANKU}

Ključne besede:

Sistem proizvodnih storitev;

Kompleksni sistem;

Robustnost;

Vrednotenje robustnosti;

Negotovost sodelovanja

*Kontaktna oseba:

cwli2008@163.com

(Li, C.W.)

614lpp@163.com

(Liang, P.P.)

Zgodovina članka:

Prejet 16. novembra 2018

Popravljen 21. aprila 2019

Sprejet 6. maja 2019 